POS PROCEEDINGS

\title{
Search for dark-photons decaying to lepton-jets with the ATLAS detector at LHC
}

\author{
Cristiano Sebastiani, on behalf of the ATLAS collaboration \\ INFN Roma and Sapienza Università di Roma \\ E-mail: cristiano.sebastianieromal.infn.it
}

\begin{abstract}
Several new physics models predict the existence of neutral particles with macroscopic life-times that decay to pairs of leptons and light hadrons with a jet-like structure (lepton-jets). These particles, decaying outside of the interaction region, will give rise to striking signatures in the detectors at the LHC. These can be detected through numerous unconventional signatures: long time-of-flight, late calorimeter energy deposits or displaced vertices. The most recent ATLAS results based on $36 \mathrm{fb}^{-1}$ of data collected in proton-proton collisions at $\sqrt{s}=13 \mathrm{TeV}$ recorded in 2015-2016 are presented. Prospects for the search for these particles with the ATLAS detector upgrade at HL-LHC are also given.
\end{abstract}

7th Annual Conference on Large Hadron Collider Physics - LHCP2019

20-25 May, 2019

Puebla, Mexico 


\section{Introduction}

Several theories beyond the Standard Model (SM) predict the existence of hidden sectors coupled to the visible one. These can provide a solution to many open questions in particle physics: dark matter, neutrino mass, matter-antimatter asymmetry and naturalness. If the two sectors are coupled through a vector portal, a dark photon $\left(\gamma_{d}\right)$ may be assumed as mediator and it may be produced at colliders. The dark photon mixing kinetically with the SM photon, depending on the structure of the hidden sector, may decay to SM particles. The kinetic mixing term $\varepsilon$ determines the lifetime, for small values the dark photon may be produced with a macroscopic life-time. The search for these long-lived particles (LLP) depends on the decay length, which can range from few $\mu \mathrm{m}$ to several meters outside the ATLAS detector [1]. LLP decaying outside of the interaction region give rise to striking signatures in the detector, representing a great challenge for both the trigger and the event reconstruction capabilities of the detector. In the study presented, only neutral long-lived particles which decay far from the interaction point are considered and therefore rely on the outermost ATLAS detectors, the hadronic calorimeter (HCAL) and the muon spectrometer (MS). The most recent ATLAS results [2] based on $36 \mathrm{fb}^{-1}$ of data collected in proton-proton collisions at $\sqrt{s}=13 \mathrm{TeV}$ recorded in 2015-2016 are presented. The new ATLAS HL-LHC setup with its huge statistics and upgraded detectors offers an opportunity to probe the yet unexplored region of the phase space. Prospects for the search for these particles with the ATLAS detector upgrade at HL-LHC are presented.

\section{Benchmark models}

The Falkowski-Ruderman-Volansky-Zupan (FRVZ) model [3] is considered as a signal benchmark for this study. This model presents a final state with two back-to-back light dark photons, with a mass of $400 \mathrm{MeV}$, produced from a SM Higgs boson decay chain together with hidden lightest stable particles. A schematic representation of the process is shown in Figure 1. The dark photons would decay via kinetic mixing to SM with the following branching fractions, which depend on its mass: $45 \%$ decay in muon pair, $45 \%$ decay in electron pair and $10 \%$ decay in pion pair.

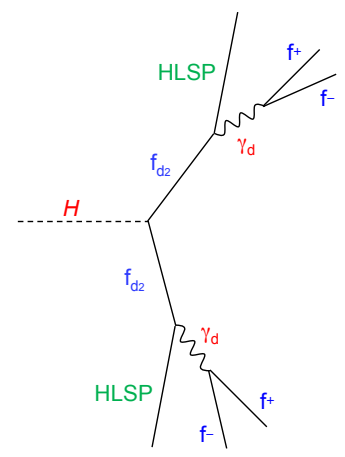

Figure 1: The $\gamma_{d}$ production together with hidden lightest stable particles (HLSP) from a Higgs hidden decay chain for the FRVZ benchmark model. 
Due to its small mass the dark photons are produced with a large boost, resulting in collimated structures containing pair of leptons and light hadrons, hereafter called lepton-jets.

\section{Lepton-jet reconstruction and event selection}

Lepton-jets are reconstructed in the ATLAS detector as cluster of displaced jets, with most of the energy deposit in the hadronic calorimeter (HCAL), and displaced muons, reconstructed in the MS with no associated tracks in the inner detector (ID). These are classified according to the number of muons and jets found within a $\Delta R$ opening cone, as shown schematically in Fig. 2:

- Muonic-LJ (TYPE-0) - At least two muons and no jets are found.

- Hadronic-LJ (TYPE-2) - A jet and no muons are found.
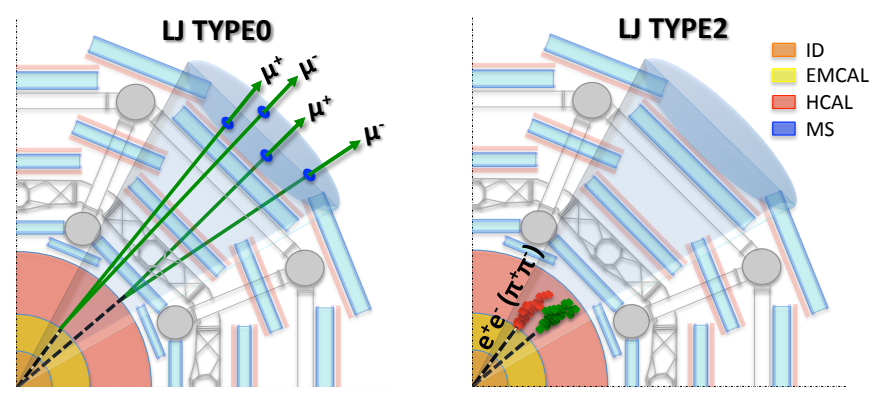

Figure 2: Schematic picture of the lepton-jet classification according to the decay final state: muons only (left) and jets only (right).

To select events with possible dark photon candidates, two dedicated triggers have been developed to select displaced objects: a multi-muon trigger that requires muons reconstructed only with information from the muon spectrometer, and a single jet trigger with a low electromagnetic fraction to select displaced jets that are produced mostly in the hadronic calorimeter.

After the trigger selection, only events with two back-to-back lepton-jets are selected and categorised in three different channels, one for each lepton-jet pair combination. To discriminate hadronic-LJ from the QCD multi-jet background and muonic-LJ from the comsic-ray background, two boosted decision trees (BDT) are used. The BDTs are trained on the following input variables:

- Muonic-LJ vs cosmic-ray

- Track parameters

- Longitudinal impact parameter

- Muon timing; arrival times measured by the trigger detectors of the MS.

- Hadronic-LJ vs multi-jet

- $E_{\mathrm{HCAL}} / E_{\mathrm{ECAL}}$; the ratio of the energy deposited in the HCAL to the energy deposited in the ECAL; 
- Jet width; the $p_{\mathrm{T}}$-weighted sum of the $\Delta R$ between each energy cluster and the jet axis;

- Jet Vertex Tagger output; a multivariate combination of track variables that are insensitive to pile-up;

- Jet timing; the energy-weighted average of the timing for each cell in the jet.

After BDT cuts, optimised to reduce the background in the signal region, the cosmic-ray background is strongly reduced and accounts only for $\sim 7 \%$ of the total background. This contribution is estimated in data collected in empty bunches, where no $p p$ collisions occur. The minor beam induced background is reduced to negligible level. The dominant QCD multi-jet background is estimated with a data-driven ABCD method. The scalar sum of the transverse momenta of the tracks reconstructed in the ID and matched to the primary vertex of the event within a $\Delta R=0.4$ cone around the LJ direction, $\sum p_{\mathrm{T}}$, and the opening azimuthal angle between the two LJs, $\Delta \Phi(L J, L J)$, are chosen as the two discriminant variables that define the ABCD matrix.

Signal lepton-jets are expected to be highly isolated in the ID and to be back-to-back, thus the signal region is defined to be at high $|\Delta \Phi|$ and low $\sum p_{\mathrm{T}}$ values.
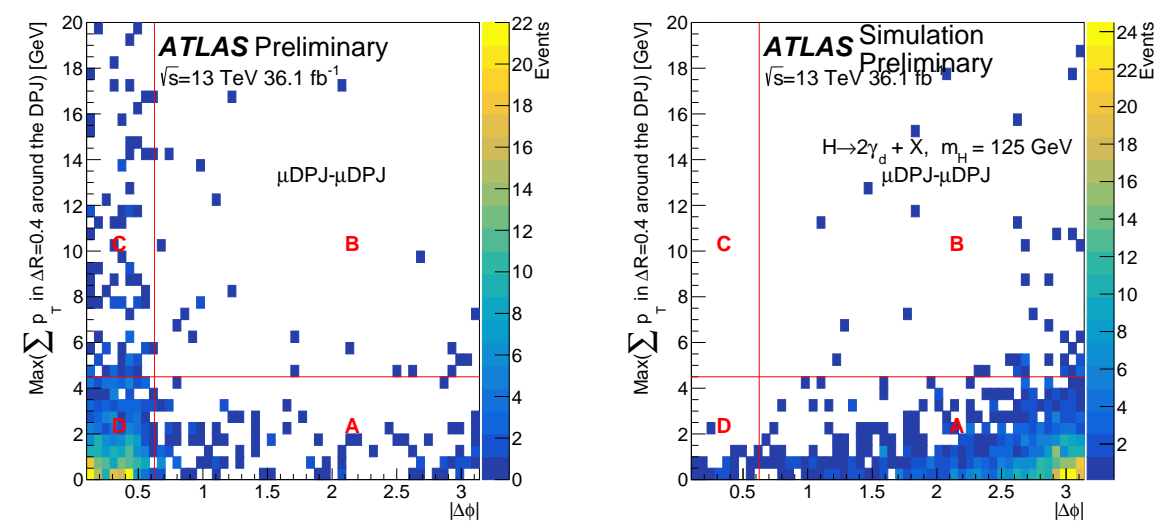

Figure 3: $A B C D$ matrix for the muonic-LJ-muonic-LJ channel in data (left) and MC signal (right) after BDT cuts. The red (solid) lines show the boundaries of the ABCD regions [2].

The distribution in the ABCD plane for the data and signal MC is shown for the muonic-LJmuonic-LJ channel in Figure 3.

\section{Results}

No excess is found over the expected background. Upper limits at 95\% CL are set on the $\sigma \times B R\left(H \rightarrow \gamma_{d} \gamma_{d}+X\right)$ as a function of the dark photon mean lifetime.

Figure 4 shows the results interpreted in the context of the FRVZ vector portal model, as contour plots in the kinetic mixing parameter $\varepsilon$ vs the dark photon mass, assuming different Higgs decay branching fractions into hidden sectors. The result is complementary to the Run-1 ATLAS search for prompt lepton-jets [5].

Prospects of the analysis sensitivity at Run-3 and HL-LHC have been estimated [6], considering a data collection of $300 \mathrm{fb}^{-1}$ and $3000 \mathrm{fb}^{-1}$ at $\sqrt{s}=14 \mathrm{TeV}$ respectively, extrapolating the muonic-LJ-muonicLJ channel results of the Run-2 search. Results are presented in Figure 5. 


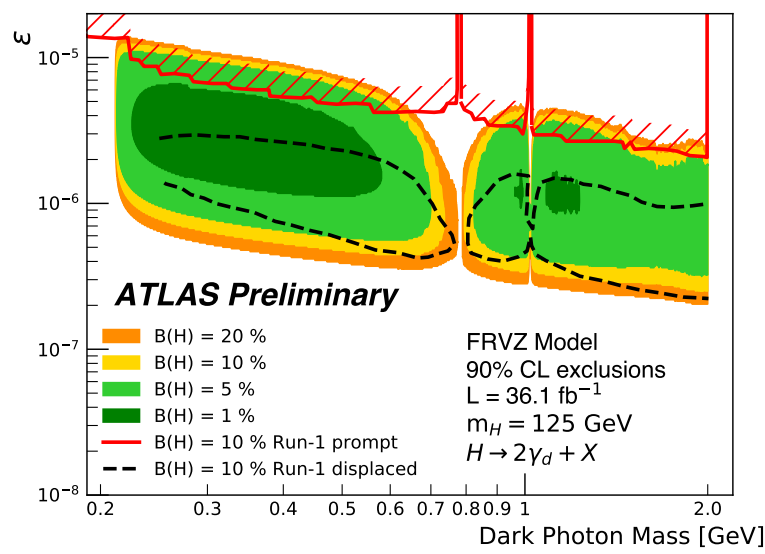

Figure 4: Parameter space exclusion plot for dark photons as a function of the $\gamma_{d}$ mass and the kinetic mixing parameter $\varepsilon$. Run-2 search for displaced lepton-jet results [2]. The figure also shows other excluded regions from ATLAS displaced [4] (black line) and prompt [5] (red line) lepton-jet searches.

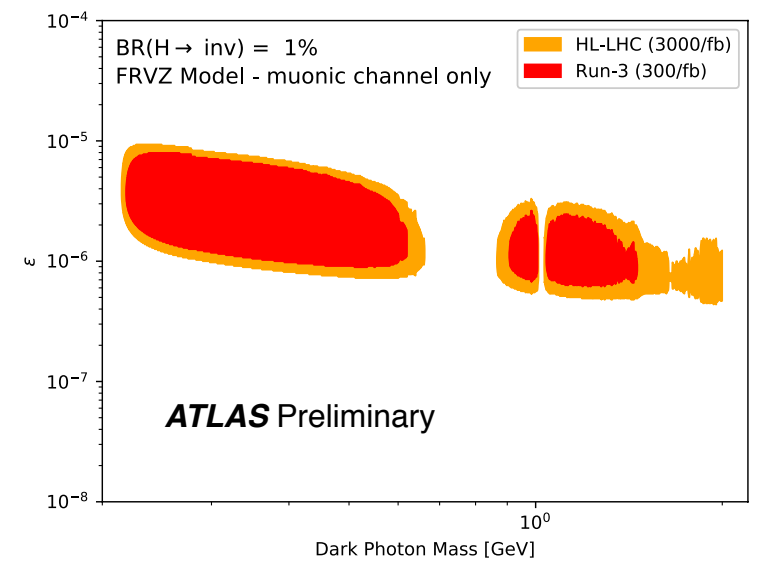

Figure 5: Parameter space exclusion plot for dark photons as a function of the $\gamma_{d}$ mass and the kinetic mixing parameter $\varepsilon$. Run-3 and HL-LHC prospects based on the muonic-LJ-muonic-LJ channel of Run-2 search for displaced lepton-jet [6].

\section{References}

[1] ATLAS Collaboration, The ATLAS Experiment at the CERN Large Hadron Collider, JINST 3 (2008) S08003.

[2] ATLAS Collaboration, "Search for light long-lived neutral particles produced in $p p$ collisions at $\sqrt{s}=13 \mathrm{TeV}$ and decaying to collimated leptons or light hadrons with the ATLAS detector." CERN-EP-2019-140, 2019.

[3] A. Falkowski, J. T. Ruderman, T. Volansky and J. Zupan, Hidden Higgs Decaying to Lepton Jets, JHEP 05 (2010) 077 [1 002 . 2952]. 
[4] ATLAS Collaboration, Search for long-lived neutral particles decaying into lepton jets in proton-proton collisions at $\sqrt{s}=8 \mathrm{TeV}$ with the ATLAS detector, JHEP 11 (2014) 088 [1409. 0746].

[5] ATLAS Collaboration, A search for prompt lepton-jets in pp collisions at $\sqrt{s}=8 \mathrm{TeV}$ with the ATLAS detector, JHEP 02 (2016) 062 [1511.05542].

[6] ATLAS Collaboration, Report on the Physics at the HL-LHC and Perspectives for the HE-LHC, 2019, 1902.10229. 